\title{
Research Article \\ Pairs of Function Spaces and Exponential Dichotomy on the Real Line
}

\author{
Adina Luminiţa Sasu \\ Department of Mathematics, Faculty of Mathematics and Computer Science, West University of Timişoara, \\ C. Coposu Boulevard. no. 4, 300223 Timişoara, Romania
}

Correspondence should be addressed to Adina Luminiţa Sasu, sasu@math.uvt.ro

Received 15 January 2010; Accepted 21 January 2010

Academic Editor: Gaston Mandata N'Guerekata

Copyright (C) 2010 Adina Luminiţa Sasu. This is an open access article distributed under the Creative Commons Attribution License, which permits unrestricted use, distribution, and reproduction in any medium, provided the original work is properly cited.

\begin{abstract}
We provide a complete diagram of the relation between the admissibility of pairs of Banach function spaces and the exponential dichotomy of evolution families on the real line. We prove that if $W \in \mathscr{H}(\mathbb{R})$ and $V \in \tau(\mathbb{R})$ are two Banach function spaces with the property that either $W \in \mathcal{W}(\mathbb{R})$ or $V \in \mathcal{U}(\mathbb{R})$, then the admissibility of the pair $(W(\mathbb{R}, X), V(\mathbb{R}, X))$ implies the existence of the exponential dichotomy. We study when the converse implication holds and show that the hypotheses on the underlying function spaces cannot be dropped and that the obtained results are the most general in this topic. Finally, our results are applied to the study of exponential dichotomy of $C_{0}$-semigroups.
\end{abstract}

\section{Introduction}

In the study of the asymptotic behavior of evolution equations the input-output conditions are very efficient tools, with wide applicability area, and give a nice connection between control theory and the qualitative theory of differential equations (see [1-16] and the reference therein). Starting with the pioneering work of Perron (see [8]) these methods were developed and improved in remarkable books (see $[1,4,6]$ ). A new and interesting perspective on this framework was proposed in [5], where the authors presented a complete study of stability, expansiveness, and dichotomy of evolution families on the half-line in terms of input-output methods. This paper was the starting point for an entire collection of studies dedicated to the input-output techniques and their applications to the qualitative theory of differential and difference equations.

If one analyzes the dichotomous properties of differential equations, then it is easily seen that there are some main technical differences between the case of evolution families 
on the half-line (see $[5,9,10])$ and the case of evolution families on the real line (see [11$16]$ ), which require a distinct analysis for each case. For instance, when one determines sufficient conditions for the existence of exponential dichotomy on the half-line, an important hypothesis is that the initial stable subspace is closed and complemented (see, e.g., [5, Theorem 4.3] or [9, Theorem 3.3]). This assumption may be dropped when we study the exponential dichotomy on the real line (see, e.g., [11, Theorem 5.1] or [16, Theorem 5.3]). These facts implicitly generate the differences between the admissibility concepts used on the real line compared with those used on the half-line and also interesting technical approaches in each case.

The aim of the present paper is to provide new and very general conditions for the existence of exponential dichotomy on the real line. We consider the problem of finding connections between the solvability of an integral equation and the existence of exponential dichotomy of evolution families on the real line. The main purpose is to obtain a complete diagram and a classification of the classes of function spaces that may be used in the study of exponential dichotomy via admissibility.

For the beginning we will present the previous results in this topic and the main objectives will be clearly specified in the context of the actual state of knowledge. We denote by $\tau(\mathbb{R})$ the class of all Banach sequence spaces $B$ which are invariant under translations, contain the continuous functions with compact support, satisfy an integral property and if $B \backslash L^{1}(\mathbb{R}, \mathbb{R}) \neq \emptyset$, then there is a continuous function $\varphi \in B \backslash L^{1}(\mathbb{R}, \mathbb{R})$. We consider $\mathscr{H}(\mathbb{R})$ the subclass of $\tau(\mathbb{R})$ satisfying the ideal property. We associate two subclasses of $\mathscr{L}(\mathbb{R})$ : $\mathcal{W}(\mathbb{R})$ - the class of all Banach function spaces with unbounded fundamental function and $\mho(\mathbb{R})$ - the class of all Banach function spaces which contain at least a nonintegrable function. A pair of function spaces $(W(\mathbb{R}, X), V(\mathbb{R}, X))$ is called admissible for an evolution family $\mathcal{U}=\{U(t, s)\}_{t \geq s}$ on the Banach space $X$ if for every test function in the input space $V(\mathbb{R}, X)$ there exists a unique solution function in the output space $W(\mathbb{R}, X)$ for the associated integral equation given by the variation of constants formula (see Definition 3.5 below).

For the first time, we have proposed in [11] a sufficient condition for exponential dichotomy, using certain Banach function spaces which are invariant under translations and we obtained the following theorem.

Theorem 1.1. If $V \in \mathcal{U}(\mathbb{R})$ and the pair $\left(C_{b}(\mathbb{R}, X), V(\mathbb{R}, X)\right)$ is admissible for an evolution family $\mathcal{U}=\{U(t, s)\}_{t \geq s}$, then $\boldsymbol{U}$ is exponentially dichotomic.

Our study has been continued and extended in [16], both for uniform dichotomy and exponential dichotomy. According to the proof of Theorem 4.8 in [16] we may give the following sufficient condition for uniform dichotomy.

Theorem 1.2. If $W \in \mathscr{H}(\mathbb{R}), V \in \tau(\mathbb{R})$, and the pair $(W(\mathbb{R}, X), V(\mathbb{R}, X))$ is admissible for an evolution family $\mathfrak{U}=\{\boldsymbol{U}(t, s)\}_{t \geq s}$, then $\mathcal{U}$ is uniformly dichotomic.

From the proof of Theorem 5.3(i) in [16] we deduce the following sufficient condition for exponential dichotomy.

Theorem 1.3. If $W \in \mathcal{W}(\mathbb{R}), V \in \tau(\mathbb{R})$, and the pair $(W(\mathbb{R}, X), V(\mathbb{R}, X))$ is admissible for an evolution family $\mathcal{U}=\{U(t, s)\}_{t \geq s}$, then $\mathcal{U}$ is exponentially dichotomic.

Taking into account the above results and their consequences, the natural question arises whether, in the general case, the output space may belong to the class $\mathscr{\ell}(\mathbb{R})$ and 
if so, which is the most general class where the input space should belong to. The aim of the present paper is to answer this question and to provide a complete study of the exponential dichotomy on the real line via integral admissibility. The answer to the above question will establish clearly how should one modify the hypotheses of Theorem 1.2 such that the admissibility of the pair $(W(\mathbb{R}, X), V(\mathbb{R}, X))$ implies the existence of the exponential dichotomy.

We will prove that if $W \in \mathscr{H}(\mathbb{R})$ and $V \in \mathcal{U}(\mathbb{R})$, then the admissibility of the pair $(W(\mathbb{R}, X), V(\mathbb{R}, X))$ is a sufficient condition for exponential dichotomy. Consequently, we will deduce a complete diagram of the study of exponential dichotomy on the real line in terms of the admissibility of function spaces (see Theorem 3.11). Specifically, if $W \in \mathscr{H}(\mathbb{R})$ and $V \in \tau(\mathbb{R})$ are two Banach function spaces with the property that either $W \in \mathcal{W}(\mathbb{R})$ or $V \in U(\mathbb{R})$, then the admissibility of the pair $(W(\mathbb{R}, X), V(\mathbb{R}, X))$ implies the existence of the exponential dichotomy. Also, in certain conditions, we deduce that the exponential dichotomy of an evolution family $\mathcal{U}=\{U(t, s)\}_{t \geq s}$ is equivalent with the admissibility of the pair $(W(\mathbb{R}, X), V(\mathbb{R}, X))$.

By an example we motivate our techniques and show that the hypotheses from our main results cannot be removed. Precisely, if $W \in \mathscr{L}(\mathbb{R})$ and $V \in \mathcal{\tau}(\mathbb{R})$ are such that $W \notin \mathcal{U}(\mathbb{R})$ and $V \notin \mho(\mathbb{R})$, then we prove that the admissibility of the pair $(W(\mathbb{R}, X), V(\mathbb{R}, X))$ does not imply the exponential dichotomy. Moreover, we show that the obtained results and their consequences are the most general in this topic.

Finally, our results are applied at the study of the exponential dichotomy of $C_{0^{-}}$ semigroups. Using function spaces which are invariant under translations, we obtain a classification of the classes of input and output spaces which may be used in the study of exponential dichotomy of semigroups in terms of input-output techniques with respect to associated integral equations.

\section{Preliminaries: Banach Function Spaces}

In this section, for the sake of clarity, we present some definitions and notations and we introduce the main classes of function spaces that will be used in our study. Let $\mathcal{M}(\mathbb{R})$ be the linear space of all Lebesgue measurable functions $u: \mathbb{R} \rightarrow \mathbb{R}$, identifying the functions equal almost everywhere.

Definition 2.1. A linear subspace $B$ of $\mathcal{M}(\mathbb{R})$ is called normed function space if there is a mapping $|\cdot|_{B}: B \rightarrow \mathbb{R}_{+}$such that

(i) $|u|_{B}=0$ if and only if $u=0$ a.e.;

(ii) $|\alpha u|_{B}=|\alpha||u|_{B}$, for all $(\alpha, u) \in \mathbb{R} \times B$;

(iii) $|u+v|_{B} \leq|u|_{B}+|v|_{B}$, for all $u, v \in B$;

(iv) if $u, v \in B$ and $|u| \leq|v|$ a.e. then $|u|_{B} \leq|v|_{B}$;

(v) if $u \in B$, then $|u| \in B$.

If $\left(B,|\cdot|_{B}\right)$ is complete, then $B$ is called Banach function space.

Definition 2.2. A Banach function space $\left(B,|\cdot|_{B}\right)$ is said to be invariant under translations if for every $(u, s) \in B \times \mathbb{R}$, the function $u_{s}: \mathbb{R} \rightarrow \mathbb{R}, u_{s}(t)=u(t-s)$ belongs to $B$ and $\left|u_{s}\right|_{B}=|u|_{B}$. 
Notations 1 . Let $C_{c}(\mathbb{R}, \mathbb{R})$ denote the linear space of all continuous functions $v: \mathbb{R} \rightarrow \mathbb{R}$ with compact support. Throughout this paper, we denote by $\tau(\mathbb{R})$ the class of all Banach function spaces $B$, which are invariant under translations, $C_{c}(\mathbb{R}, \mathbb{R}) \subset B$, and satisfy the following conditions:

(i) for every $t>s$ there is $\alpha(t, s)>0$ such that $\int_{s}^{t}|u(\tau)| d \tau \leq \alpha(t, s)|u|_{B}$, for all $u \in B$;

(ii) if $B \backslash L^{1}(\mathbb{R}, \mathbb{R}) \neq \emptyset$ then there is a continuous function $\varphi \in B \backslash L^{1}(\mathbb{R}, \mathbb{R})$.

For examples of Banach function spaces from the class $\tau(\mathbb{R})$ we refer to [11].

Let $\mathscr{\ell}(\mathbb{R})$ be the class of all Banach function spaces $B \in \tau(\mathbb{R})$ with the property that if $|u| \leq|v|$ a.e. and $v \in B$, then $u \in B$.

For every $A \subset \mathbb{R}$ we denote by $\chi_{A}$ the characteristic function of the set $A$. Then, if $B \in \mathscr{H}(\mathbb{R})$, we have that $\chi_{[a, b)} \in B$, for every $a, b \in \mathbb{R}$ with $a<b$.

Definition 2.3. Let $B \in \mathscr{H}(\mathbb{R})$. The mapping $F_{B}:(0, \infty) \rightarrow \mathbb{R}, F_{B}(t)=\left|X_{[0, t)}\right|_{B}$ is called the fundamental function of the space $B$.

For the proof of the next proposition we refer to [16, Proposition 2.8].

Proposition 2.4. Let $B \in \mathscr{d}(\mathbb{R})$ and $v>0$. If $u: \mathbb{R} \rightarrow \mathbb{R}_{+}$is a function, which belongs to $B$ and with the property that $q_{u}: \mathbb{R} \rightarrow \mathbb{R}_{+}, q_{u}(t)=\int_{t}^{t+1} u(s) d$ s belongs to $B$, then the functions

$$
f_{u}, g_{u}: \mathbb{R} \longrightarrow \mathbb{R}_{+}, \quad f_{u}(t)=\int_{-\infty}^{t} e^{-v(t-s)} u(s) d s, \quad g_{u}(t)=\int_{t}^{\infty} e^{-v(s-t)} u(s) d s
$$

belong to $B$.

Example 2.5. Let $M^{1}(\mathbb{R}, \mathbb{R})$ be the linear space of all $u \in \mathcal{M}(\mathbb{R})$ with the property that $\sup _{t \in \mathbb{R}} \int_{t}^{t+1}|u(s)| d s<\infty$. With respect to the norm

$$
\|u\|_{M^{1}}:=\sup _{t \in \mathbb{R}} \int_{t}^{t+1}|u(s)| d s
$$

this is a Banach function space which belongs to $\mathscr{H}(\mathbb{R})$.

Lemma 2.6. If $B \in \tau(\mathbb{R})$, then $B \subset M^{1}(\mathbb{R}, \mathbb{R})$.

Proof. Let $\alpha>0$ be such that $\int_{0}^{1}|u(\tau)| d \tau \leq \alpha|u|_{B}$, for all $u \in B$. Then, we have that

$$
\int_{t}^{t+1}|v(\tau)| d \tau=\int_{0}^{1}\left|v_{t}(\tau)\right| d \tau \leq \alpha\left|v_{t}\right|_{B}=\alpha|v|_{B}, \quad \forall t \in \mathbb{R}, \forall v \in B
$$


Notations 2. In what follows we denote by

(i) $\mathcal{W}(\mathbb{R})$ the class of all Banach function spaces $B \in \mathscr{\ell}(\mathbb{R})$ with $\sup _{t>0} F_{B}(t)=\infty$;

(ii) $\mho(\mathbb{R})$ the class of all Banach function spaces $B \in \tau(\mathbb{R})$ with the property that $B \backslash$ $L^{1}(\mathbb{R}, \mathbb{R}) \neq \emptyset$

(iii) $\mathcal{O}(\mathbb{R})$ the class of all Banach function spaces $B \in \mathscr{\ell}(\mathbb{R})$ with the property that for every $u: \mathbb{R} \rightarrow \mathbb{R}_{+}$in $B$, the function $q_{u}: \mathbb{R} \rightarrow \mathbb{R}_{+}, q_{u}(t)=\int_{t}^{t+1} u(s) d s$ belongs to $B$.

Remark 2.7. (i) For examples of Banach function spaces from the class $\mathcal{W}(\mathbb{R})$ we refer to $[16$, Proposition 2.9].

(ii) If $B \in \mathcal{U}(\mathbb{R})$ then there is a continuous function $\varphi: \mathbb{R} \rightarrow \mathbb{R}_{+}$with $\varphi \in B \backslash L^{1}(\mathbb{R}, \mathbb{R})$.

Notation 1. Let $C_{0}(\mathbb{R}, \mathbb{R})$ be the space of all continuous functions $v: \mathbb{R} \rightarrow \mathbb{R}$ with $\lim _{t \rightarrow \pm \infty} v(t)=0$, which is Banach space with respect to the norm $|\|v\||\left|:=\sup _{t \in \mathbb{R}}\right| v(t) \mid$.

Lemma 2.8. Let $B$ be a Banach function space with $B \in \mathscr{H}(\mathbb{R}) \backslash \mathcal{W}(\mathbb{R})$. Then $C_{0}(\mathbb{R}, \mathbb{R}) \subset B$.

Proof. Let $L:=\sup _{t>0} F_{B}(t)$. Let $v \in C_{0}(\mathbb{R}, \mathbb{R})$. Then there is an unbounded increasing sequence $\left(t_{n}\right) \subset(0, \infty)$ such that $|v(t)| \leq 1 /(n+1)$, for all $|t| \geq t_{n}$ and all $n \in \mathbb{N}$. Setting $v_{n}=v_{X\left[-t_{n}, t_{n}\right]}$ we have that

$$
\left|v_{n+p}-v_{n}\right|_{B} \leq \frac{1}{n+1}\left(\left|X_{\left[-t_{n+p},-t_{n}\right)}\right|_{B}+\left|\chi_{\left(t_{n}, t_{n+p}\right]}\right|_{B}\right) \leq \frac{2 L}{n+1}, \quad \forall p \in \mathbb{N}^{*}, \forall n \in \mathbb{N}
$$

From the above inequality we deduce that $\left(v_{n}\right)$ is fundamental in the Banach space $B$, so there is $w \in B$ such that $v_{n} \rightarrow w$ in $B$. According to [16, Lemma 2.4] there is a subsequence $\left(v_{k_{n}}\right)$ such that $v_{k_{n}} \rightarrow w$ a.e. This implies that $v \equiv w$ a.e., so $v=w$ in $B$. Thus $v \in B$ and the proof is complete.

Notation 2. Let $X$ be a real or complex Banach space. For every $B \in \tau(\mathbb{R})$ we denote by $B(\mathbb{R}, X)$ the linear space of all Bochner measurable functions $v: \mathbb{R} \rightarrow X$ with the property that the mapping $N_{v}: \mathbb{R} \rightarrow \mathbb{R}_{+}, N_{v}(t)=\|v(t)\|$ lies in $B$. With respect to the norm $\|v\|_{B(\mathbb{R}, X)}:=$ $\left|N_{v}\right|_{B}, B(\mathbb{R}, X)$ is a Banach space.

\section{Exponential Dichotomy for Evolution Families on the Real Line}

Let $X$ be a real or complex Banach space. The norm on $X$ and on $\mathcal{B}(X)$, the Banach algebra of all bounded linear operators on $X$, will be denoted by $\|\cdot\|$. Denote by $I_{d}$ the identity operator on $X$. First, we remind some basic definitions.

Definition 3.1. A family $\mathfrak{U}=\{U(t, s)\}_{t \geq s}$ of bounded linear operators on $X$ is called an evolution family if the following properties hold:

(i) $U\left(t_{0}, t_{0}\right)=I_{d}$ and $U(t, s) U\left(s, t_{0}\right)=U\left(t, t_{0}\right)$, for all $t \geq s \geq t_{0}$;

(ii) for every $x \in X$ and every $t_{0} \in \mathbb{R}$ the mapping $t \mapsto U\left(t, t_{0}\right) x$ is continuous on $\left[t_{0}, \infty\right)$ and the mapping $s \mapsto U\left(t_{0}, s\right) x$ is continuous on $\left(-\infty, t_{0}\right]$;

(iii) there are $M \geq 1$ and $\omega>0$ such that $\left\|U\left(t, t_{0}\right)\right\| \leq M e^{\omega\left(t-t_{0}\right)}$, for all $t \geq t_{0}$. 
Definition 3.2. An evolution family $u=\{U(t, s)\}_{t \geq s}$ is said to be uniformly dichotomic if there are a family of projections $\{P(t)\}_{t \in \mathbb{R}}$ and a constant $K \geq 1$ such that

(i) $U\left(t, t_{0}\right) P\left(t_{0}\right)=P(t) U\left(t, t_{0}\right)$, for all $t \geq t_{0}$;

(ii) the restriction $U\left(t, t_{0}\right)_{\mid}: \operatorname{ker} P\left(t_{0}\right) \rightarrow \operatorname{ker} P(t)$ is an isomorphism, for all $t \geq t_{0}$;

(iii) $\left\|U\left(t, t_{0}\right) x\right\| \leq K\|x\|$, for all $x \in \operatorname{Im} P\left(t_{0}\right)$ and all $t \geq t_{0}$;

(iv) $\left\|U\left(t, t_{0}\right) y\right\| \geq(1 / K)\|y\|$, for all $y \in$ ker $P\left(t_{0}\right)$ and all $t \geq t_{0}$.

Definition 3.3. An evolution family $\mathcal{U}=\{U(t, s)\}_{t \geq s}$ is said to be exponentially dichotomic if there exist a family of projections $\{P(t)\}_{t \in \mathbb{R}}$ and two constants $K \geq 1$ and $v>0$ such that

(i) $U\left(t, t_{0}\right) P\left(t_{0}\right)=P(t) U\left(t, t_{0}\right)$, for all $t \geq t_{0}$ ；

(ii) the restriction $U\left(t, t_{0}\right)_{\mid}: \operatorname{ker} P\left(t_{0}\right) \rightarrow \operatorname{ker} P(t)$ is an isomorphism, for all $t \geq t_{0}$;

(iii) $\left\|U\left(t, t_{0}\right) x\right\| \leq K e^{-v\left(t-t_{0}\right)}\|x\|$, for all $x \in \operatorname{Im} P\left(t_{0}\right)$ and all $t \geq t_{0}$;

(iv) $\left\|U\left(t, t_{0}\right) y\right\| \geq(1 / K) e^{v\left(t-t_{0}\right)}\|y\|$, for all $y \in$ ker $P\left(t_{0}\right)$ and all $t \geq t_{0}$.

Remark 3.4. It is obvious that if an evolution family is exponentially dichotomic, then it is uniformly dichotomic.

One of the most efficient tool in the study of the dichotomic behavior of an evolution family is represented by the so-called input-output techniques. The input-output method considered in this paper is the admissibility of a pair of function spaces. Indeed, let $W, V$ be two Banach function spaces such that $W \in \mathscr{H}(\mathbb{R})$ and $V \in \mathcal{\tau}(\mathbb{R})$.

Definition 3.5. The pair $(W(\mathbb{R}, X), V(\mathbb{R}, X))$ is said to be admissible for $\mathcal{U}$ if for every $v \in$ $V(\mathbb{R}, X)$ there exists a unique $f \in W(\mathbb{R}, X)$ such that the pair $(f, v)$ satisfies the equation

$$
f(t)=U(t, s) f(s)+\int_{s}^{t} U(t, \tau) v(\tau) d \tau, \quad \forall t \geq s
$$

Remark 3.6. If the pair $(W(\mathbb{R}, X), V(\mathbb{R}, X))$ is admissible for $\mathcal{U}$, then it makes sense to define the operator $Q: V(\mathbb{R}, X) \rightarrow W(\mathbb{R}, X), Q(v)=f$, where $f \in W(\mathbb{R}, X)$ is such that the pair $(f, v)$ satisfies $\left(E_{\mathfrak{U}}\right)$. Then $Q$ is a bounded linear operator (see [16, Proposition 4.4]).

Let $\mathcal{U}=\{U(t, s)\}_{t \geq s}$ be an evolution family on $X$ and $W \in \mathscr{H}(\mathbb{R})$. For every $t \in \mathbb{R}$, we consider the stable subspace $X_{s}(t)$ as the space of all $x \in X$ with the property that the function

$$
\delta_{x}: \mathbb{R} \longrightarrow X, \quad \delta_{x}(\tau)= \begin{cases}U(\tau, t) x, & \tau \geq t \\ 0, & \tau<t\end{cases}
$$

belongs to $W(\mathbb{R}, X)$ and we define the unstable subspace $X_{u}(t)$ as the space of all $x \in X$ with the property that there is a function $\varphi_{x} \in W(\mathbb{R}, X)$ such that $\varphi_{x}(t)=x$ and $\varphi_{x}(\tau)=U(\tau, s) \varphi_{x}(s)$, for all $s \leq \tau \leq t$.

An important information concerning the structure of the projection family associated with a uniformly dichotomic evolution family was obtained in [16, Theorem 4.8] and this is given by the following. 
Theorem 3.7. Let $\mathcal{U}=\{U(t, s)\}_{t \geq s}$ be an evolution family on $X$ and let $W, V$ be two Banach function spaces with $W \in \mathscr{H}(\mathbb{R})$ and $V \in \mathcal{Z}(\mathbb{R})$. If the pair $(W(\mathbb{R}, X), V(\mathbb{R}, X))$ is admissible for the evolution family $\mathcal{U}$, then $\mathcal{U}$ is uniformly dichotomic with respect to the family of projections $\{P(t)\}_{t \in \mathbb{R}}$, where

$$
\operatorname{Im} P(t)=X_{s}(t), \quad \operatorname{ker} P(t)=X_{u}(t), \quad \forall t \in \mathbb{R} .
$$

Taking into account the results obtained in $[11,16]$, an interesting open question is whether in the study of exponential dichotomy, the output space may belong to the general class $\mathscr{H}(\mathbb{R})$. To answer this question, the first purpose of this paper is to prove the following theorem.

Theorem 3.8. Let $\mathcal{U}=\{U(t, s)\}_{t \geq s}$ be an evolution family on the Banach space $X$ and let $W, V$ be two Banach function spaces with $W \in \mathscr{H}(\mathbb{R})$ and $V \in \mathcal{U}(\mathbb{R})$. If the pair $(W(\mathbb{R}, X), V(\mathbb{R}, X))$ is admissible for $\mathfrak{U}$, then $\boldsymbol{U}$ is uniformly exponentially dichotomic. results.

The proof will be constructive and therefore, we will present several intermediate

Theorem 3.9. Let $\mathcal{U}=\{U(t, s)\}_{t \geq s}$ be an evolution family on the Banach space $X$ and let $W, V$ be two Banach function spaces with $W \in \mathscr{H}(\mathbb{R})$ and $V \in \mathcal{U}(\mathbb{R})$. If the pair $(W(\mathbb{R}, X), V(\mathbb{R}, X))$ is admissible for $\mathcal{U}$, then there are $K, v>0$ such that

$$
\left\|U\left(t, t_{0}\right) x\right\| \leq K e^{-v\left(t-t_{0}\right)}\|x\|, \quad \forall t \geq t_{0}, \forall x \in X_{s}\left(t_{0}\right)
$$

Proof. According to Theorem 3.7 and Definition 3.2(iii) we have that there is $\lambda>0$ such that

$$
\left\|U\left(t, t_{0}\right) x\right\| \leq \lambda\|x\|, \quad \forall t \geq t_{0}, \forall x \in X_{s}\left(t_{0}\right) .
$$

Since $V \in \mathcal{U}\left(\mathbb{R}_{+}\right)$, from Remark 2.7(ii) we have that there is a continuous function $\varphi: \mathbb{R} \rightarrow \mathbb{R}_{+}$ with $\varphi \in V \backslash L^{1}(\mathbb{R}, \mathbb{R})$. Using the invariance under translations of the space $V$, we may assume that there is $r>1$ such that

$$
\int_{0}^{r} \varphi(s) d s \geq 2 \frac{e \lambda^{2}\|Q\| \|\left.\varphi\right|_{V}}{F_{W}(1)}
$$

Since $\int_{1 / n}^{r-1 / n} \varphi(s) d s \underset{n \rightarrow \infty}{\rightarrow} \int_{0}^{r} \varphi(s) d s$ there is $n_{0} \in \mathbb{N}^{*}$ such that

$$
\int_{1 / n_{0}}^{r-1 / n_{0}} \varphi(s) d s \geq \frac{1}{2} \int_{0}^{r} \varphi(s) d s .
$$

Let $\alpha: \mathbb{R} \rightarrow[0,1]$ be a continuous function with supp $\alpha \subset(0, r)$ and $\alpha(t)=1$, for $t \in$ $\left[1 / n_{0}, r-1 / n_{0}\right]$. Then, the function $\psi: \mathbb{R} \rightarrow \mathbb{R}_{+}, \psi(t)=\alpha(t) \varphi(t)$ is continuous and from (3.5) and (3.6) we have that

$$
\int_{0}^{r} \psi(t) d t \geq \int_{1 / n_{0}}^{r-1 / n_{0}} \varphi(t) d t \geq \frac{e \lambda^{2}\|Q\||\varphi|_{V}}{F_{W}(1)} .
$$


Let $t_{0} \in \mathbb{R}$ and let $x \in X_{s}\left(t_{0}\right)$. We consider the functions

$$
\begin{gathered}
v: \mathbb{R} \rightarrow X, \quad v(t)=\psi\left(t-t_{0}\right) U\left(t, t_{0}\right) x, \\
f: \mathbb{R} \rightarrow X, \quad f(t)= \begin{cases}\int_{t_{0}}^{t} \psi\left(\tau-t_{0}\right) d \tau U\left(t, t_{0}\right) x, & t \geq t_{0}, \\
0, & t<t_{0} .\end{cases}
\end{gathered}
$$

Since $v \in C_{c}(\mathbb{R}, X)$ it follows that $v \in V(\mathbb{R}, X)$. Setting $q=\int_{0}^{r} \psi(\tau) d \tau$ we observe that $f(t)=q U\left(t, t_{0}\right) x$, for all $t \geq t_{0}+r$. Since $x \in X_{s}\left(t_{0}\right)$ we deduce that $f \in W(\mathbb{R}, X)$. A simple computation shows that the pair $(f, v)$ satisfies $\left(E_{\mathfrak{U}}\right)$, so $f=Q(v)$. This implies that

$$
\|f\|_{W(\mathbb{R}, X)} \leq\|Q\|\|v\|_{V(\mathbb{R}, X)} .
$$

According to relation (3.4) we observe that

$$
\|v(t)\|=\psi\left(t-t_{0}\right)\left\|U\left(t, t_{0}\right) x\right\| \leq \lambda\|x\| \psi_{t_{0}}(t), \quad \forall t \in \mathbb{R},
$$

and using the invariance under translations of the space $V$ we deduce that $\|v\|_{V(\mathbb{R}, X)} \leq$ $\left.\lambda\|x\||| \psi\right|_{V}$. From $\psi(t) \leq \varphi(t)$, for all $t \in \mathbb{R}$, we have that $|\psi|_{V} \leq|\varphi|_{V}$. Thus we obtain that

$$
\|v\|_{V(\mathbb{R}, X)} \leq \lambda\|x\||\varphi|_{V}
$$

From $\left\|U\left(t_{0}+r+1, t_{0}\right) x\right\| \leq \lambda\left\|U\left(\tau, t_{0}\right) x\right\|=(\lambda / q)\|f(\tau)\|$, for all $\tau \in\left[t_{0}+r, t_{0}+r+1\right]$, we have that $\left\|U\left(t_{0}+r+1, t_{0}\right) x\right\|_{X\left[t_{0}+r, t_{0}+r+1\right)}(\tau) \leq(\lambda / q)\|f(\tau)\|$, for all $\tau \in \mathbb{R}$, which implies that

$$
F_{W}(1)\left\|U\left(t_{0}+r+1, t_{0}\right) x\right\| \leq \frac{\lambda}{q}\|f\|_{W(\mathbb{R}, X)} .
$$

Setting $l=r+1$, from relations (3.10)-(3.13) it follows that

$$
q F_{W}(1)\left\|U\left(t_{0}+l, t_{0}\right) x\right\| \leq \lambda^{2}\|Q\||\varphi|_{V}\|x\|
$$

Using relations (3.7) and (3.14) we deduce that $\left\|U\left(t_{0}+l, t_{0}\right) x\right\| \leq(1 / e)\|x\|$. Taking into account that $l$ does not depend on $t_{0}$ or $x$, we have that

$$
\left\|U\left(t_{0}+l, t_{0}\right) x\right\| \leq \frac{1}{e}\|x\|, \quad \forall t_{0} \in \mathbb{R}, \forall x \in X_{s}\left(t_{0}\right) .
$$

Let $v=1 / l$ and $K=\lambda e$. Let $t \geq t_{0}$ and $x \in X_{s}\left(t_{0}\right)$. Then, there are $j \in \mathbb{N}$ and $s \in[0, l)$ such that $t=t_{0}+j l+s$. Using relations (3.4) and (3.15) we obtain that $\left\|U\left(t, t_{0}\right) x\right\| \leq \lambda\left\|U\left(t_{0}+j l, t_{0}\right) x\right\| \leq$ $\lambda e^{-j}\|x\| \leq K e^{-v\left(t-t_{0}\right)}\|x\|$, which completes the proof. 
Theorem 3.10. Let $\mathcal{U}=\{U(t, s)\}_{t \geq s}$ be an evolution family on the Banach space $X$ and let $W, V$ be two Banach function spaces with $W \in \mathscr{H}(\mathbb{R})$ and $V \in \mathcal{U}(\mathbb{R})$. If the pair $(W(\mathbb{R}, X), V(\mathbb{R}, X))$ is admissible for $\mathcal{U}$, then there are $K, v>0$ such that

$$
\left\|U\left(t, t_{0}\right) x\right\| \geq \frac{1}{K} e^{\nu\left(t-t_{0}\right)}\|x\|, \quad \forall t \geq t_{0}, \forall x \in X_{u}\left(t_{0}\right) .
$$

Proof. Let $M \geq 1$ and $\omega>0$ be given by Definition 3.1. According to Theorem 3.7 and Definition 3.2(iv) we have that there is $\lambda>0$ such that

$$
\left\|U\left(t, t_{0}\right) x\right\| \geq \frac{1}{\lambda}\|x\|, \quad \forall t \geq t_{0}, \forall x \in X_{u}\left(t_{0}\right)
$$

Since $V \in \mathcal{U}\left(\mathbb{R}_{+}\right)$, from Remark 2.7(ii) we have that there is a continuous function $\varphi: \mathbb{R} \rightarrow \mathbb{R}_{+}$ with $\varphi \in V \backslash L^{1}(\mathbb{R}, \mathbb{R})$. Using the invariance under translations of the space $V$ we may assume that there is $r>1$ such that

$$
\int_{0}^{r} \varphi(s) d s \geq 2 \frac{\lambda M e^{\omega+1}\|Q\||\varphi|_{V}}{F_{W}(1)}
$$

Using similar arguments with those in the proof of Theorem 3.9 we obtain that there is a continuous function $\psi: \mathbb{R} \rightarrow \mathbb{R}_{+}$with supp $\psi \subset(0, r), \psi(t) \leq \varphi(t)$, for all $t \in \mathbb{R}$ and

$$
\int_{0}^{r} \psi(\tau) d \tau \geq \frac{\lambda M e^{\omega+1}\|Q\||\varphi|_{V}}{F_{W}(1)}
$$

Let $t_{0} \in \mathbb{R}$ and $x \in X_{u}\left(t_{0}\right)$. Then, there is $\varphi_{x} \in W(\mathbb{R}, X)$ such that $\varphi_{x}\left(t_{0}\right)=x$ and $\varphi_{x}(\tau)=$ $U(\tau, s) \varphi_{x}(s)$, for all $s \leq \tau \leq t_{0}$. We consider the functions

$$
\begin{gathered}
v: \mathbb{R} \longrightarrow X, \quad v(t)=-\psi\left(t-t_{0}\right) U\left(t, t_{0}\right) x, \\
f: \mathbb{R} \longrightarrow X, \quad f(t)= \begin{cases}\int_{t}^{\infty} \psi\left(\tau-t_{0}\right) d \tau U\left(t, t_{0}\right) x, & t \geq t_{0}, \\
q \varphi_{x}(t), & t<t_{0},\end{cases}
\end{gathered}
$$

where $q=\int_{0}^{r} \psi(\tau) d \tau$. We have that $v \in C_{c}(\mathbb{R}, X)$, so $v \in V(\mathbb{R}, X)$. Using relation (3.17) we have that

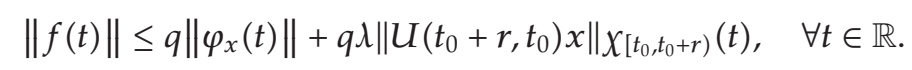

From this inequality, since $W \in \mathscr{\ell}(\mathbb{R})$ we deduce that $f \in W(\mathbb{R}, X)$. An easy computation shows that the pair $(f, v)$ satisfies $\left(E_{\mathfrak{U}}\right)$, so $f=Q(v)$. Then, we have that

$$
\|f\|_{W(\mathbb{R}, X)} \leq\|Q\|\|v\|_{V(\mathbb{R}, X)} .
$$


Using relation (3.17) we have that

$$
\|v(t)\|=\psi\left(t-t_{0}\right)\left\|U\left(t, t_{0}\right) x\right\| \leq \psi\left(t-t_{0}\right) \lambda\left\|U\left(t_{0}+r, t_{0}\right) x\right\|, \quad \forall t \in \mathbb{R}
$$

which implies that

$$
\|v\|_{V(\mathbb{R}, X)} \leq|\psi|_{V} \lambda\left\|U\left(t_{0}+r, t_{0}\right) x\right\| \leq|\varphi|_{V} \lambda\left\|U\left(t_{0}+r, t_{0}\right) x\right\| .
$$

Since $x=\varphi_{x}\left(t_{0}\right)=U\left(t_{0}, t\right) \varphi_{x}(t)$, for all $t \in\left[t_{0}-1, t_{0}\right]$, we deduce that

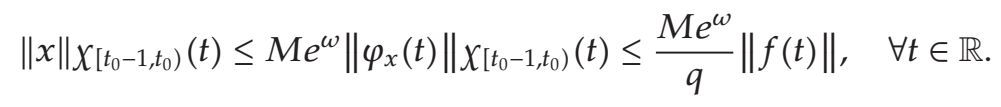

This shows that

$$
q\|x\| F_{W}(1) \leq M e^{\omega}\|f\|_{W(\mathbb{R}, X)} .
$$

From relations (3.19)-(3.27) it follows that $\left\|U\left(t_{0}+r, t_{0}\right) x\right\| \geq e\|x\|$. Taking into account that $r$ does not depend on $t_{0}$ or $x$, we have that

$$
\left\|U\left(t_{0}+r, t_{0}\right) x\right\| \geq e\|x\|, \quad \forall t_{0} \in \mathbb{R}, \forall x \in X_{u}\left(t_{0}\right) .
$$

We set $v=1 / r$ and $K=\lambda e$. Let $t \geq t_{0}$ and $x \in X_{u}\left(t_{0}\right)$. Then, there are $j \in \mathbb{N}$ and $s \in[0, r)$ such that $t=t_{0}+j r+s$. Using relations (3.17) and (3.28) we obtain that $\left\|U\left(t, t_{0}\right) x\right\| \geq(1 / \lambda) \| U\left(t_{0}+\right.$ $\left.j r, t_{0}\right) x\left\|\geq(1 / \lambda) e^{j}\right\| x\left\|\geq(1 / K) e^{v\left(t-t_{0}\right)}\right\| x \|$, which completes the proof.

Now, we may give the proof of Theorem 3.8.

Proof of Theorem 3.8. This immediately follows from Theorems 3.7, 3.9, and 3.10.

Now, we may give the main result of the paper, which establishes a complete diagram concerning the study of exponential dichotomy on the real line in terms of integral admissibility.

Theorem 3.11. Let $\mathcal{U}=\{U(t, s)\}_{t \geq s}$ be an evolution family on the Banach space $X$ and let $W, V$ be two Banach function spaces with $W \in \mathscr{H}(\mathbb{R})$ and $V \in \mathcal{\tau}(\mathbb{R})$. If $W \in \mathcal{W}(\mathbb{R})$ or $V \in \mathcal{U}(\mathbb{R})$, then the following assertions hold:

(i) if the pair $(W(\mathbb{R}, X), V(\mathbb{R}, X))$ is admissible for $\boldsymbol{U}$, then $\boldsymbol{U}$ is uniformly exponentially dichotomic;

(ii) if $V \subset W$ and one of the spaces $V, W$ belongs to the class $\mathcal{O}(\mathbb{R})$, then $U$ is exponentially dichotomic if and only if the pair $(W(\mathbb{R}, X), V(\mathbb{R}, X))$ is admissible for $\mathcal{u}$.

Proof. (i) This follows from Theorems 1.3 and 3.8.

(ii) Necessity. Suppose that $\mathcal{U}$ is exponentially dichotomic with respect to the family of projections $\{P(t)\}_{t \in \mathbb{R}}$ and the constants $K, v>0$. Then, we have that $L:=\sup _{t \in \mathbb{R}}\|P(t)\|<\infty$ (see, e.g., [13]). 
Let $v \in V(\mathbb{R}, X)$. We consider the function

$$
f: \mathbb{R} \longrightarrow X, \quad f(t)=\int_{-\infty}^{t} U(t, s) P(s) v(s) d s-\int_{t}^{\infty} U(s, t)_{\mid}^{-1}(I-P(s)) v(s) d s,
$$

where for every $s>t, U(s, t)_{\mid}^{-1}$ denotes the inverse of the operator $U(s, t) \mid \operatorname{ker} P(t) \rightarrow$ ker $P(s)$.

If $V \in \mathcal{O}(\mathbb{R})$, then using Proposition 2.4 we obtain that $f \in V(\mathbb{R}, X)$. Since $V \subset W$ we deduce that $f \in W(\mathbb{R}, X)$.

If $W \in \mathcal{O}(\mathbb{R})$ then, since $V \subset W$ we have that $v \in W(\mathbb{R}, X)$. Using Proposition 2.4 it follows that $f \in W(\mathbb{R}, X)$.

An easy computation shows that the pair $(f, v)$ satisfies $\left(E_{\mathfrak{U}}\right)$. The uniqueness of $f$ is immediate (see, e.g., [16, the Necessity part of Theorem 5.3]). In conclusion, the pair $(W(\mathbb{R}, X), V(\mathbb{R}, X))$ is admissible for the evolution family $\mathcal{U}$.

The natural question arises whether the hypotheses from Theorem 3.11 can be dropped and also if the conditions given by this theorem are the most general in this topic. The answers are given by the following example.

Example 3.12. Let $W \in \mathscr{H}(\mathbb{R})$ and $V \in \tau(\mathbb{R})$ be such that $W \notin \mathcal{W}(\mathbb{R})$ and $V \notin \mho(\mathbb{R})$. Then $V \subset L^{1}(\mathbb{R}, \mathbb{R})$ and according to Lemma 2.8 we have that $C_{0}(\mathbb{R}, \mathbb{R}) \subset W$.

We consider the function

$$
\varphi: \mathbb{R} \longrightarrow \mathbb{R}, \quad \varphi(t)= \begin{cases}\frac{1}{t+1}, & t \geq 0 \\ 1-t, & t<0 .\end{cases}
$$

Then $\varphi$ is a decreasing function.

Let $X=\mathbb{R}^{2}$ endowed with the norm $\left\|\left(x_{1}, x_{2}\right)\right\|:=\left|x_{1}\right|+\left|x_{2}\right|$, for all $\left(x_{1}, x_{2}\right) \in \mathbb{R}^{2}$. For every $t \geq s$ we consider the operator

$$
U(t, s): X \longrightarrow X, \quad U(t, s)\left(x_{1}, x_{2}\right)=\left(\frac{\varphi(t)}{\varphi(s)} x_{1}, e^{t-s} x_{2}\right)
$$

Then $\mathcal{U}=\{U(t, s)\}_{t \geq s}$ is an evolution family on $X$.

We prove that the pair $(W(\mathbb{R}, X), V(\mathbb{R}, X))$ is admissible for $\mathcal{U}$. Let $v=\left(v_{1}, v_{2}\right) \in$ $V(\mathbb{R}, X)$. Then $v_{1}, v_{2} \in L^{1}(\mathbb{R}, \mathbb{R})$. We consider the function

$$
f: \mathbb{R} \longrightarrow X, \quad f(t)=\left(\int_{-\infty}^{t} \frac{\varphi(t)}{\varphi(\tau)} v_{1}(\tau) d \tau,-\int_{t}^{\infty} e^{-(\tau-t)} v_{2}(\tau) d \tau\right)
$$


We have that $f$ is correctly defined and an easy computation shows that the pair $(f, v)$ satisfies $\left(E_{\mathfrak{U}}\right)$. We set

$$
f_{1}(t)=\int_{-\infty}^{t} \frac{\varphi(t)}{\varphi(s)} v_{1}(s) d \tau, \quad f_{2}(t)=-\int_{t}^{\infty} e^{-(\tau-t)} v_{2}(\tau) d \tau, \quad \forall t \in \mathbb{R}
$$

We prove that $f_{1} \in C_{0}(\mathbb{R}, \mathbb{R})$. Since $v_{1} \in L^{1}(\mathbb{R}, \mathbb{R})$, from

$$
\left|f_{1}(t)\right| \leq \int_{-\infty}^{t}\left|v_{1}(\tau)\right| d \tau, \quad \forall t \in \mathbb{R}
$$

we have that $\lim _{t \rightarrow-\infty} f_{1}(t)=0$. Let $\varepsilon>0$. Then, there is $\delta>0$ such that $\int_{\delta}^{\infty}\left|v_{1}(\tau)\right| d \tau<\varepsilon$. It follows that

$$
\left|f_{1}(t)\right| \leq \frac{\varphi(t)}{\varphi(\delta)} \int_{-\infty}^{\delta} \frac{\varphi(\delta)}{\varphi(\tau)}\left|v_{1}(\tau)\right| d \tau+\int_{\delta}^{t}\left|v_{1}(\tau)\right| d \tau<\frac{\delta+1}{t+1}\left\|v_{1}\right\|_{1}+\varepsilon, \quad \forall t \geq \delta .
$$

The above inequality implies that $\varlimsup_{t \rightarrow \infty}\left|f_{1}(t)\right| \leq \varepsilon$. Since $\varepsilon>0$ was arbitrary we obtain that there exists $\lim _{t \rightarrow \infty} f_{1}(t)=0$. Using similar arguments with those in (3.34) we deduce that $\lim _{t \rightarrow \infty} f_{2}(t)=0$.

Let $\varepsilon>0$. Then there is $\gamma<0$ such that $\int_{-\infty}^{\gamma}\left|v_{2}(\tau)\right| d \tau<\varepsilon$. It follows that

$$
\left|f_{2}(t)\right| \leq e^{t-\gamma} \int_{\gamma}^{\infty} e^{\gamma-\tau}\left|v_{2}(\tau)\right| d \tau+\int_{t}^{\gamma}\left|v_{2}(\tau)\right| d \tau<e^{t-\gamma}\left\|v_{2}\right\|_{1}+\varepsilon, \quad \forall t \leq \gamma
$$

From this inequality we have that $\varlimsup_{t \rightarrow-\infty}\left|f_{2}(t)\right| \leq \varepsilon$. Since $\varepsilon>0$ was arbitrary, it follows that there exists $\lim _{t \rightarrow-\infty} f_{2}(t)=0$. Thus, we deduce that $f \in C_{0}(\mathbb{R}, X)$, so $f \in W(\mathbb{R}, X)$.

To prove the uniqueness of $f$, let $\tilde{f} \in W(\mathbb{R}, X)$ be such that the pair $(\tilde{f}, v)$ satisfies $\left(E_{\mathcal{U}}\right)$. Setting $g=\tilde{f}-f$ we have that $g \in W(\mathbb{R}, X)$ and $g(t)=U(t, s) g(s)$, for all $t \geq s$. If $g=\left(g_{1}, g_{2}\right)$, then we deduce that

$$
\begin{gathered}
g_{1}(t)=\frac{\varphi(t)}{\varphi(s)} g_{1}(s), \quad \forall t \geq s, \\
g_{2}(t)=e^{t-s} g_{2}(s), \quad \forall t \geq s .
\end{gathered}
$$

Let $t \in \mathbb{R}$. Using Lemma 2.6 and integrating in (3.37) we have that

$$
\left|g_{1}(t)\right|=\int_{s}^{s+1} \frac{\varphi(t)}{\varphi(\tau)}\left|g_{1}(\tau)\right| d \tau \leq \frac{\varphi(t)}{\varphi(s+1)}\|g\|_{M_{1}(\mathbb{R}, X)} \quad \forall s \leq t-1
$$

For $s \rightarrow-\infty$ in (3.39) we obtain that $g_{1}(t)=0$. 
From relation (3.38) we have that

$$
g_{2}(t)=e^{-(\tau-t)} g_{2}(\tau), \quad \forall \tau \geq t
$$

Integrating in relation (3.40) on $[\tau, \tau+1]$ and using Lemma 2.6, we deduce that

$$
\left|g_{2}(t)\right|=\int_{\tau}^{\tau+1} e^{-(\xi-t)}\left|g_{2}(\xi)\right| d \xi \leq e^{-(\tau-t)}\|g\|_{M_{1}(\mathbb{R}, X)^{\prime}} \quad \forall \tau \geq t
$$

For $\tau \rightarrow \infty$ in (3.41) it follows that $g_{2}(t)=0$. Since $t \in \mathbb{R}$ was arbitrary we have that $g=0$, so $\tilde{f}=f$. Thus, the pair $(W(\mathbb{R}, X), V(\mathbb{R}, X))$ is admissible for the evolution family $u$.

Suppose that $\mathcal{U}$ is exponentially dichotomic with respect to the family of projections $\{P(t)\}_{t \in \mathbb{R}}$ and the constants $K, v>0$. According to [13, Proposition 3.1] we have that $\operatorname{Im} P(t)=$ $\left\{x \in X: \sup _{\tau \geq t}\|U(\tau, t) x\|<\infty\right\}$, which implies that $\operatorname{Im} P(t)=\mathbb{R} \times\{0\}$, for all $t \in \mathbb{R}$. Then, we obtain that

$$
\left|\frac{\varphi(t)}{\varphi(s)} x_{1}\right| \leq K e^{-v(t-s)}\left|x_{1}\right|, \quad \forall t \geq s, \forall x_{1} \in \mathbb{R}
$$

or equivalently

$$
\frac{\varphi(t)}{\varphi(s)} \leq K e^{-v(t-s)}, \quad \forall t \geq s
$$

which is absurd. In conclusion, the pair $(W(\mathbb{R}, X), V(\mathbb{R}, X))$ is admissible for $\mathcal{U}$, but, for all that, the evolution family $\mathcal{U}$ is not exponentially dichotomic.

\section{Applications to the Case of $C_{0}$-Semigroups}

In this section, by applying the central theorems from the Section 3 we deduce several consequences of the main results for the study of exponential dichotomy of $C_{0}$-semigroups. Let $X$ be a real or complex Banach space.

Definition 4.1. A family $\mathbf{T}=\{T(t)\}_{t \geq 0}$ of bounded linear operators on $X$ is said to be a $C_{0^{-}}$ semigroup if the following properties are satisfied:

(i) $T(0)=I_{d}$, the identity operator on $X$;

(ii) $T(t+s)=T(t) T(s)$, for all $t, s \geq 0$;

(iii) $\lim _{t \searrow 0} T(t) x=x$, for every $x \in X$. 
Definition 4.2. A $C_{0}$-semigroup $\mathbf{T}=\{T(t)\}_{t \geq 0}$ is said to be exponentially dichotomic if there exist a projection $P \in B(X)$ and two constants $K \geq 1$ and $v>0$ such that

(i) $P T(t)=T(t) P$, for all $t \geq 0$;

(ii) $\|T(t) x\| \leq K e^{-v t}\|x\|$, for all $x \in \operatorname{Im} P$ and all $t \geq 0$;

(iii) $\|T(t) x\| \geq(1 / K) e^{\nu t}\|x\|$, for all $x \in \operatorname{ker} P$ and all $t \geq 0$;

(iv) the restriction $T(t)_{\mid}: \operatorname{ker} P \rightarrow \operatorname{ker} P$ is an isomorphism, for every $t \geq 0$.

Remark 4.3. (i) If $\mathbf{T}=\{T(t)\}_{t \geq 0}$ is a $C_{0}$-semigroup, we can associate to $\mathbf{T}$ an evolution family $\mathcal{U}_{T}=\left\{U_{T}(t, s)\right\}_{t \geq s}$, by $U_{T}(t, s)=T(t-s)$, for every $t \geq s$.

(ii) A $C_{0}$-semigroup $\mathbf{T}=\{T(t)\}_{t \geq 0}$ is exponentially dichotomic if and only if the associated evolution family $\mathfrak{U}_{T}=\left\{U_{T}(t, s)\right\}_{t>S}$ is exponentially dichotomic (see [12, Proposition 4.4]).

Let $W, V$ be two Banach function spaces such that $W \in \mathscr{\ell}(\mathbb{R})$ and $V \in \tau(\mathbb{R})$.

Definition 4.4. The pair $(W(\mathbb{R}, X), V(\mathbb{R}, X))$ is said to be admissible for the $C_{0}$-semigroup $\mathbf{T}=$ $\{T(t)\}_{t \geq 0}$ if for every $v \in V(\mathbb{R}, X)$ there is a unique $f \in W(\mathbb{R}, X)$ such that

$$
f(t)=T(t-s) f(s)+\int_{s}^{t} T(t-\tau) v(\tau) d \tau, \quad \forall t \geq s .
$$

Theorem 4.5. Let $T=\{T(t)\}_{t \geq 0}$ be a $C_{0}$-semigroup on the Banach space $X$ and let $W, V$ be two Banach function spaces with $W \in \mathscr{H}(\mathbb{R})$ and $V \in \tau(\mathbb{R})$. If $W \in \mathcal{W}(\mathbb{R})$ or $V \in \mathcal{U}(\mathbb{R})$, then the following assertions hold:

(i) if the pair $(W(\mathbb{R}, X), V(\mathbb{R}, X))$ is admissible for $\mathbf{T}$, then $\mathbf{T}$ is uniformly exponentially dichotomic;

(ii) if $V \subset W$ and one of the spaces $V, W$ belongs to the class $\mathcal{O}(\mathbb{R})$, then $\mathbf{T}$ is exponentially dichotomic if and only if the pair $(W(\mathbb{R}, X), V(\mathbb{R}, X))$ is admissible for $\mathbf{T}$.

Proof. This follows from Theorem 3.11 and Remark 4.3(ii).

Remark 4.6. According to the example given in the previous section we deduce that the hypothesis $W \in \mathcal{W}(\mathbb{R})$ or $V \in \mathcal{U}(\mathbb{R})$ cannot be removed. Moreover, Theorem 4.5 provides a complete answer concerning the study of exponential dichotomy of semigroups using inputoutput techniques with respect to the associated integral equation.

\section{Acknowledgment}

This work is supported by the Exploratory Research Project PN 2 ID 1081 code 550/2009. 


\section{References}

[1] J. L. Daleckii and M. G. Krein, Stability of Solutions of Differential Equations in Banach Space, vol. 4 of Translations of Mathematical Monographs, American Mathematical Society, Providence, RI, USA, 1974.

[2] J. H. Liu, G. M. N'Guérékata, and N. Van Minh, Topics on Stability and Periodicity in Abstract Differential Equations, vol. 6 of Series on Concrete and Applicable Mathematics, World Scientific, Hackensack, NJ, USA, 2008.

[3] Q. Liu, N. Van Minh, G. Nguerekata, and R. Yuan, "Massera type theorems for abstract functional differential equations," Funkcialaj Ekvacioj, vol. 51, no. 3, pp. 329-350, 2008.

[4] J. L. Massera and J. J. Schäffer, Linear Differential Equations and Function Spaces, vol. 21 of Pure and Applied Mathematics, Academic Press, New York, NY, USA, 1966.

[5] N. Van Minh, F. Räbiger, and R. Schnaubelt, "Exponential stability, exponential expansiveness, and exponential dichotomy of evolution equations on the half-line," Integral Equations and Operator Theory, vol. 32, no. 3, pp. 332-353, 1998.

[6] N. Van Minh, G. M. N'Guérékata, and R. Yuan, Lectures on the Asymptotic Behavior of Solutions of Differential Equations, Nova Science, New York, NY, USA, 2008.

[7] A. Pazy, Semigroups of Linear Operators and Applications to Partial Differential Equations, vol. 44 of Applied Mathematical Sciences, Springer, New York, NY, USA, 1983.

[8] O. Perron, "Die Stabilitätsfrage bei Differentialgleichungen," Mathematische Zeitschrift, vol. 32, no. 1, pp. 703-728, 1930.

[9] B. Sasu and A. L. Sasu, "Exponential dichotomy and $\left(l^{p}, l^{q}\right)$-admissibility on the half-line," Journal of Mathematical Analysis and Applications, vol. 316, no. 2, pp. 397-408, 2006.

[10] B. Sasu, "Uniform dichotomy and exponential dichotomy of evolution families on the half-line," Journal of Mathematical Analysis and Applications, vol. 323, no. 2, pp. 1465-1478, 2006.

[11] A. L. Sasu and B. Sasu, "Exponential dichotomy on the real line and admissibility of function spaces," Integral Equations and Operator Theory, vol. 54, no. 1, pp. 113-130, 2006.

[12] A. L. Sasu, "Exponential dichotomy for evolution families on the real line," Abstract and Applied Analysis, vol. 2006, Article ID 31641, 6 pages, 2006.

[13] A. L. Sasu and B. Sasu, "Exponential dichotomy and admissibility for evolution families on the real line," Dynamics of Continuous, Discrete E Impulsive Systems. Series A, vol. 13, no. 1, pp. 1-26, 2006.

[14] A. L. Sasu and B. Sasu, "Discrete admissibility, $l^{p}$-spaces and exponential dichotomy on the real line," Dynamics of Continuous, Discrete E Impulsive Systems. Series A, vol. 13, no. 5, pp. 551-561, 2006.

[15] B. Sasu and A. L. Sasu, "Exponential trichotomy and $p$-admissibility for evolution families on the real line," Mathematische Zeitschrift, vol. 253, no. 3, pp. 515-536, 2006.

[16] A. L. Sasu, "Integral equations on function spaces and dichotomy on the real line," Integral Equations and Operator Theory, vol. 58, no. 1, pp. 133-152, 2007. 